\title{
Treatment of Scapular Dyskinesis with Reflexive Neuromuscular Stimulation: A Case Report
}

\author{
Alli Z. Powell, DAT, AT 1 \& Russell T. Baker, PhD, DAT, $A T^{2}$
}

'Colorado Mesa University, Grand Junction, $\mathrm{CO}$; ${ }^{2}$ University of Idaho, Moscow, ID

\section{ABSTRACT}

Scapular dyskinesis is an abnormal movement of the scapula due to poor motor control of the surrounding musculature and can lead to other glenohumeral pathologies. The pathology results in the lateral tilting of the scapula during many glenohumeral joint movements and weight-bearing activities of the upper extremity (i.e., plank). The typical conservative treatment protocol focuses on strengthening surrounding musculature and is often a lengthy protocol. A weakness of strengthening protocols is the failure to address the motor function of targeted muscles at the level of the central nervous system (CNS) to restore dynamic stability and motor control. Reactive Neuromuscular Stabilization (RNS) is a novel treatment that targets the CNS to address motor control impairments to restore the normal muscular and joint stability and function. Purpose: The purpose of this case study was to demonstrate the efficacy and the outcomes of using RNS as a treatment for dysfunctions such as scapular dyskinesis. A 20-year-old female intercollegiate swimmer presented with significant mid-back pain that failed to resolve without treatment. The patient was diagnosed with scapular dyskinesis as well as presented with a number of postural concerns, trigger points, and pain with multiple activities. The initial protocol for treatment for this patient was a standard conservative treatment protocol focused on strengthening. After six weeks of treatment without improvement, the clinician modified care to include RNS. Following three treatments across seven days, the patient's symptoms decreased significantly and the patient met discharge criteria. At an eleven-month follow-up, the patient's improvements were maintained. The patient in this case report demonstrates the effectiveness of RNS while treating scapular dyskinesis and the importance of recognizing the cause of the dysfunction early within the evaluation.

\section{Key Phrases}

Diagnostic testing and physical examination, upper extremity, patient-reported outcomes, manual techniques

\section{Correspondence}

Dr. Alli Powell, Colorado Mesa University, 1100 North Ave. Grand Junction, CO.

E-mail: azeigel@gmail.com

\section{Full Citation}

Powell A \& Rusty BT. Treatment of Scapular Dyskinesis with Reflexive Neuromuscular Stimulation: A Case Report. Clin Pract Athl Train. 2019;2(3):35-47.

https://doi.org/2019/0003.3.

Submitted: February 21, 2019 Accepted: October 21, 2019

\section{INTRODUCTION}

Scapular dyskinesis (SD) is defined as irregular

motion of the scapula. ${ }^{1-2}$ Scapular dyskinesis is often evaluated visually by the clinician from a posterior view with the patient performing active glenohumeral $(\mathrm{GH})$ motions. ${ }^{1-5}$ The scapula provides stability to the $\mathrm{GH}$ articulation through the contraction of the surrounding muscles to centralize the rotation of the $\mathrm{GH}$ joint. ${ }^{1-2}$ The position of the scapula adjusts to overhead activity by moving in the following motions: protraction, retraction, elevation, depression, and rotation. When the scapula fails to move synergistically with the GH joint, compensatory and dysfunctional movement patterns are created.' Most abnormal movement patterns of the scapula have been suggested to result from poor functioning of the stabilizing muscles of the scapula. 1,3 Increased protraction at the medial border of the scapula during $\mathrm{GH}$ motion, such as horizontal adduction, flexion, and abduction are some of the most common signs of SD. ${ }^{3}$ The abnormal movement, when not caused by long thoracic nerve damage, is typically thought to result from hyper-activation of the upper trapezius in conjunction with decreased activation of the lower trapezius and serratus anterior. 6 Though nerve damage to the long thoracic and spinal accessory nerves may result in decreased muscular function, the commonality of these pathologies as the cause of the dyskinesis is less than 5\%.' Other common causes include thoracic kyphosis, clavicular fracture nonunion, high-grade AC joint instability, or soft tissue inflexibility. ${ }^{2}$

Conservative treatment is the most commonly recommended course of action in cases of SD; 
however, if nerve damage is present, surgical intervention may be indicated. ${ }^{4}$ Most nonoperative rehabilitation includes mobility exercises of the thoracic spine and shoulder (e.g., stretching, joint mobilizations, thoracic mobility exercises), closed and open chain strengthening exercises (e.g., push up plus, serratus anterior ceiling punches), and other reactive exercises (e.g., catching and throwing) targeting the improvement of motor control. A few concerns with conservative rehabilitation of SD is lengthy treatment protocols (i.e. weeks to months); and a primary focus on strengthening only the surrounding musculature of the shoulder girdle instead of focusing on neuromuscular reeducation and the restoration of dynamic stability and functional movement patterns. ${ }^{7}$

As a proposed cause for SD is poor motor control and a loss of dynamic stability, along with over activation of related musculature, a neuromuscular re-patterning technique such as Reflexive Neuromuscular Stabilization (RNS) seems appropriate. This technique was derived from a treatment called Reactive Neuromuscular Training (RNT). The concept of RNT is to restore dynamic stability and motor control post-injury with rehabilitation techniques which target the central nervous system (CNS). As the CNS reacts to a stimulus to create joint stability, there is a conversion from conscious thought to unconscious. ${ }^{8}$ Rather than cue the patient verbally and have discussions regarding what they are doing incorrectly, the goal of RNT is to exacerbate the dysfunctional movement with an external stimulus to bring a more clear perception to the patient of the error that is occurring. ${ }^{9}$ When using RNT, a clinician applies an external stimulus to the patient's body to promote an unconscious response by the CNS to produce the appropriate motor response and correct the faulty movement pattern. ${ }^{10}$ Where RNS deviates from RNT is modification to make the process more 'reflexive' than 'reactive' to enhance the unconscious and reflexive response that a physically active patient needs to produce during activity to

Copyright ( $)$ by Indiana State University All rights reserved. ISSN Online 2577-8188 maintain dynamic stability. ${ }^{11}$ Let us take for an example, when a patient performs a squat they have a significant valgus collapse. When using RNT we may utilize an elastic band around the patient's knees as a constant force that they are pushing against as they squat to force the patient to engage appropriate knee and hip stabilizers throughout the movement. In contrast, when switching to an RNS mindset of treatment, the clinician would apply the valgus force at the patient's knees with varying amounts of force and at unexpected times to force the patient's reflexes to initiate and correct the faulty movement pattern with their reflex to the stimulus. Again, the largest difference is having a constant and known force they are reacting to versus and changing and unexpected force their reflexes must engage in to correct the movement pattern.

Currently, there are no studies regarding the application of RNS and SD and only a few studies have been published on the application of RNS or RNT; thus, little is known about the application of these techniques in patient care. Therefore, the purpose of this case study was to report the outcomes of incorporating RNS into the rehabilitation program of an intercollegiate swimmer diagnosed with SD who had failed to improve using traditional conservative methods. The patient gave informed consent to participate in the study as well as was informed that the data collected would be submitted for publication. This study was approved by the Institutional Review Board.

\section{PATIENT INFORMATION}

\section{Patient}

The patient was a 20 -year-old female Division I intercollegiate swimmer who presented with right upper back pain and no known mechanism of injury. She described experiencing her current pain for approximately six months. Her pain was isolated to the right medial border of the scapula with a gradual onset and no known 
mechanism of injury. When the symptoms initially began, her primary complaint was experiencing pain at the end of inhalation. When the pain started, the patient was home for the summer (i.e. between spring and fall semesters) and used self-treatment consisting of three months of complete rest from activity and occasional superficial heat without resolution of her symptoms. The patient reported to the Athletic Training clinic after four months of unresolved symptoms for the start of the fall semester.

The initial examination revealed pain along the medial aspect of the right scapula during inhalation, while sitting in good posture, or while wearing a backpack. The patient did not reveal any red flags for cancer, chronic illnesses, or family history of illnesses. She had experienced pain in the upper right back eight months previously when she was lifting weights, however, the pain resolved without treatment. She reported the current episode as "feeling different" and unrelated to the current condition. She was not taking any medication for the discomfort and had no other reports of treatment. Her reported worst pain on the Numerical Pain Rating Scale (NPRS) was a seven out of ten when sitting erect and at the end of an inhalation. A zero on the NPRS is classified as no pain, whereas a ten is classified as the worst pain imaginable. ${ }^{12}$ Disability was measured using the Disablement in the Physically Active (DPA) Scale, which is scored from zero (no disability) to 64 (maximum disability). ${ }^{13-14}$ The patient reported a disability score of 28 on the DPA Scale on the initial evaluation. The Patient Specific Functional Scale (PSFS) was utilized to identify activities within her daily life that were causing pain. This scale utilizes a score between zero (cannot perform) to ten (no problem performing). ${ }^{15}$ Her three primary painful activities were breathing ( 4 out of 10), sitting up straight (4 out of 10) and wearing a backpack (6 out of 10).

Copyright (C) by Indiana State University All rights reserved. ISSN Online 2577-8188
The examination did not reveal signs of inflammation, ecchymosis, or deformities surrounding the area of pain. Her natural posture was forward head, forward shoulder, and increased kyphosis. The postures were exacerbated in a seated position. In a seated position, visual evaluation of the patient's breathing revealed all of the motion for inhalation stemming from the chest rather than from the diaphragm. To test breathing functionality, the clinician used a modification of the Manual Assessment of Respiratory Motion (MARM) test. ${ }^{16}$ The modification of the MARM test was done via palpation and observation to assess the 3-Dimensional movement of the trunk and chest during inhalation and exhalation. The clinician placed their hands along the patient's mid to low back with the thumbs parallel to the spine and fingers splaying laterally (Figure 1). While the patient completed normal inspiration/expiration, the clinician felt for the motion of breathing to either be lateral, superior, anterior, and/or posterior. A normal pattern consists of lateral, anterior, posterior, and limited superior motion. ${ }^{17}$ This patient revealed a primary upward motion in breathing, with absent posterior and lateral motions with inhalation.

Tender points (TPs) at the middle portion of the insertion of the rhomboid minor, superior portion of the insertion of the rhomboid major, middle

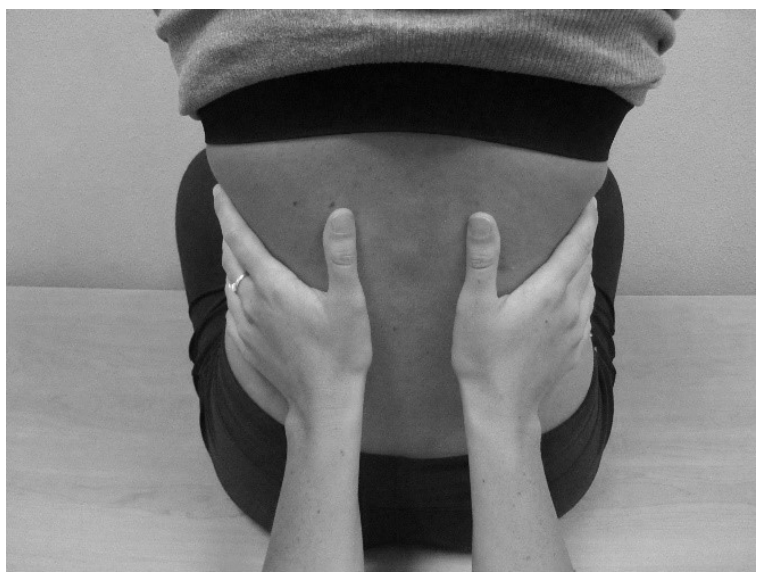

Figure 1. Hand Positioning for Modified MARM Test 
trapezius superior portion of the insertion and superolateral muscle belly, and serratus posterior superolateral muscle belly were identified with palpation. Differentiation of the musculature was determined through active contractions of the muscles. In addition to reporting TPs, the patient stated that she generally felt "tighter" on the right medial aspect of the scapula, compared bilaterally. The patient also reported tenderness to palpation at the first and second ribs with a superior to inferior pressure posterior to the clacivle and on the posterior aspect of the eleventh and twelfth ribs in a supine position.

Range of motion testing was performed and revealed no limitations or pain with any of the following active range of motions (AROM) at the shoulder: flexion, extension, internal rotation (IR) at a 90-90 position, external rotation (ER) at a 90-90 position, abduction, horizontal adduction, or horizontal abduction. Observation during AROM testing revealed the patient had substantial SD on the right side that was most prominent with flexion, abduction, and horizontal adduction (Figure 2). The following passive ranges of motion (PROM) at the shoulder were equal bilaterally, within normal limits, and nonpainful: flexion, extension, IR, ER, horizontal

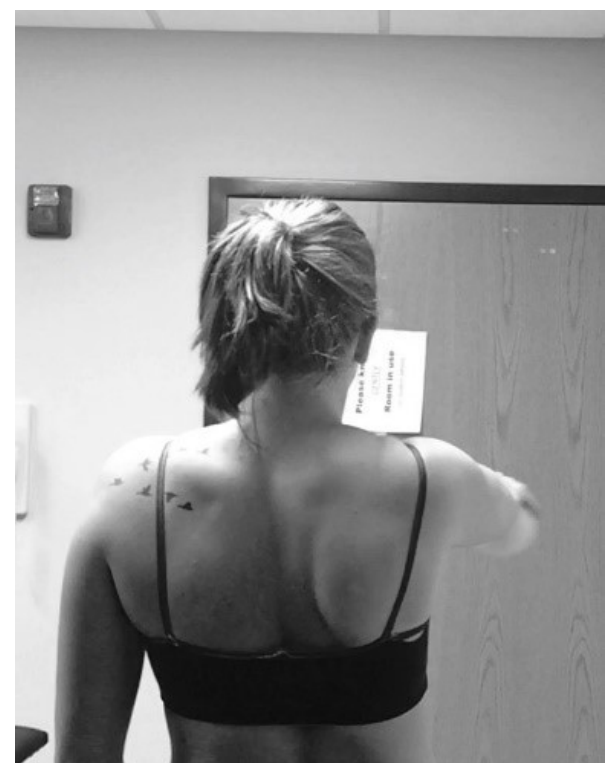

Figure 2. Scapular Dyskinesis PreTreatment

Copyright (C) by Indiana State University 38 All rights reserved. ISSN Online 2577-8188 adduction, abduction and horizontal abduction. Strength testing of the rotator cuff muscles, deltoid, pectoralis major, biceps brachii, and triceps brachii were all 5/5 and non-painful when compared bilaterally. The right rhomboids had decreased strength, $4 / 5$, when compared bilaterally.

The patient displayed a positive sulcus sign bilaterally and SD on the right when in a push up plus position both in non-weight bearing and weight bearing positions. Based on the lateral scapula slide test (LSST), the patient met the established threshold, $1.5 \mathrm{~cm}$ to be a positive test, of difference during 90 degrees of abduction and was $.2 \mathrm{~cm}$ and $.3 \mathrm{~cm}$ from the threshold in the positions with hands on hips and at 90 degrees horizontal adduction while standing to indicate SD (Table 1).1,18-19 Each measurement was taken from the spinous process even with the inferior angle of the scapula for each motion. The LSST was performed with the patient's hands by her side, progressed to hands on the hips, and 90 degrees of abduction. Though the LSST is designed exclusively for those three motions, the clinician also measured the differences when the patient completed horizontal adduction. The Apprehension and Relocation, Empty Can, and Gerber Lift Off tests were negative.

Neurological screening and function was within normal limits. Based upon these findings, the patient was classified with right SD and conservative rehabilitation was initiated without limitation in activity levels.

\section{INTERVENTION}

The first six weeks of treatment and rehabilitation consisted of a combination of moist heat packs (MHP), Positional Release Therapy (PRT), breathing retraining, Primal Reflex Release Techniques (PRRT), instrument assisted soft tissue mobilization (IASTM), massage, strengthening, and stretching. Treatment sessions began with MHPs to stimulate blood flow to the affected area, and to help increase patient relaxation and comfort. After the application of 
Table 1. Measurements Taken in the Scapular Slide Test $(\mathrm{cm})$

\begin{tabular}{lcccc}
\hline & Left & Right & Left & Right \\
\hline Arm Position & \multicolumn{2}{c}{ Day 1} & \multicolumn{2}{c}{ Day 3 } \\
\cline { 2 - 6 } Down by sides & 9.5 & 9.5 & 9.5 & 9.5 \\
Hands on hips & 8.9 & 10.2 & 8.9 & 8.9 \\
90 degrees abduction & 10.2 & 7.6 & 10.2 & 10.2 \\
90 degrees horizontal adduction & 12.7 & 14 & 12.7 & 12.7 \\
\hline
\end{tabular}

the MHPs, PRT was used to release the TPs present during the initial evaluation. Following PRT, breathing retraining exercises were used to restore normal diaphragmatic breathing. The techniques utilized were a combination of breathing exercise developed by Michael Grant White ${ }^{20}$ and PPRT techniques developed by John lams, and were selected because of positive clinical outcomes experienced by the treating clinician during previous patient care..$^{21}$ The breathing exercise was similar to the traditional "clam shell" exercise for hip external rotator strengthening (Figure 3 and Figure 4). However, the breathing component required the patient to attempt maximal exhalation (i.e., "blow all your air out") and then move through full hip external rotation with the top leg while holding their breath throughout the motion. Once the knees returned to the starting position, the patient was cued to inhale. The length of the count varied by the patient's ability to hold her breath. When the patient returned to the starting position, the required response was to have the patient take a "gasping" breath, meaning the patient felt as

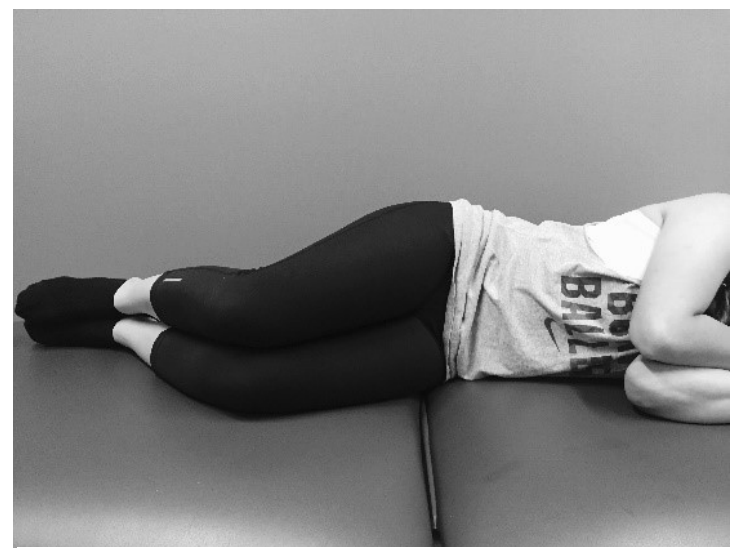

Figure 3. Starting and Ending Position of the "Clam Shell" Exercise if she could not hold her breath any longer, thus taking a large reflexive inhalation.

The PRRT technique was then used to address the patient reported rhomboid tightness. Initially, a facilitation technique was used on the rhomboids due to the forward shoulder posture of the patient; however, the technique did not produce improvement, so the clinician then inhibited the rhomboids in an attempt to decrease pain and tightness. Next, IASTM and massage were used to reduce remaining TPs on the affected side rhomboid major, rhomboid minor, upper and middle trapezius, and serratus posterior, as well as to restore function and ROM. Therapeutic exercises were used to improve muscular control and strength of the serratus anterior and lower trapezius muscles to improve scapular stabilization within a functional movement pattern (Table 2). Finally, a stretching regimen was used to release tight anterior musculature and improve posture.

Over the first six weeks of therapy, the patient would complete this therapy protocol 1 time per

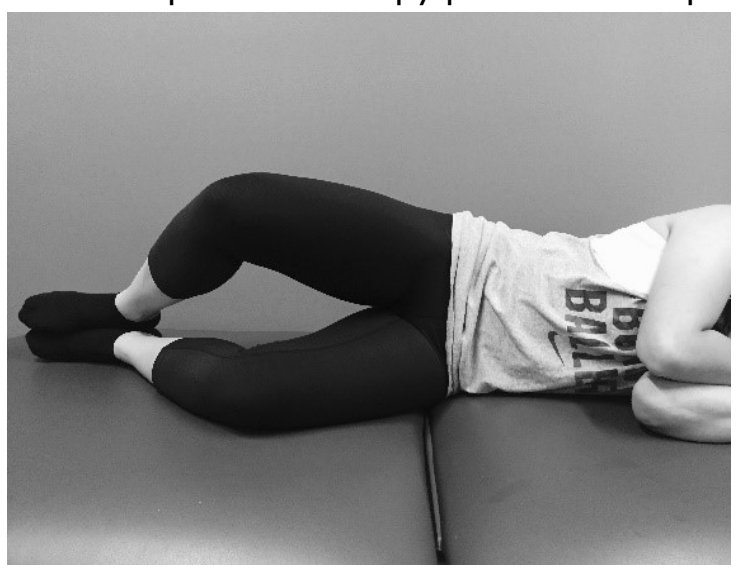

Figure 4. "Clam Shell" Exercise Motion 
Table 2. Frequency and Duration of Each Rehabilitative Exercise Performed

\begin{tabular}{lccc}
\hline Exercise & $\begin{array}{c}\text { Times per } \\
\text { Week* }\end{array}$ & $\begin{array}{c}\text { Weeks } \\
\text { Performed }\end{array}$ & Duration \\
\hline Low row/ scapula pinches with a red resistance band & $2-3$ & 2 & $3 \times 8-10$ \\
Supine Scapular Retraction & $1-5$ & 2 & $2 \times 8$ \\
$\begin{array}{l}\text { Standing Scapular Retraction Against Exercise Ball } \\
\text { Scapular control exercises (patient holding a weighted ball }\end{array}$ & $1-5$ & 3 & $2 \times 10$ \\
$\begin{array}{l}\text { and moving into flexion, horizontal adduction, horizontal } \\
\text { abduction \& abduction) }\end{array}$ & $2-3$ & 4 & $3 \times 30$ seconds \\
$\begin{array}{l}\text { Push up plus on BOSU } \\
\text { l's, Y's, T's }\end{array}$ & 2 & 3.5 & $3 \times 8$ \\
Horizontal adduction with 3lb weighted ball with RNS & 2 & 3.5 & $2 \times 8$ \\
\hline
\end{tabular}

*Times per week varied weekly based on availability and travel

day and four days per week, on average.

During this time, the patient reported short-term pain relief and TP reduction. The patient typically reported a decrease in pain following each treatment session; yet, the pain and TPs returned without any substantial improvement by the end of a two-hour swim practice or by her next visit. When a treatment was provided prior to practice, the patient reported a resolution of her complaint, but it would only remain resolved through 50 to $75 \%$ of the practice period ( 2 hours). Treatment provided on non-training days followed a similar pattern, but usually increased the duration of her pain resolution from approximately 90 minutes to 3 hours. During these six weeks, discernible improvements in the patient's strength and dyskinesis were recorded (Table 4). Due to the lack of consistent and long-lasting patient-reported or disease-oriented improvement, the clinician re-evaluated the patient and decided to add RNS to her established rehabilitation protocol. The clinical reasoning for this choice focused on the belief the patient's functional motor patterns (i.e., stability) were not being addressed at the subconscious level, and a more reflexive neuromuscular intervention was needed to normalize movement patterns and postures at the subconscious level.

Copyright (C) by Indiana State University All rights reserved. ISSN Online 2577-8188
The initial treatment goal for utilizing RNS was to decrease SD during standing horizontal adduction because this was the most difficult movement for the patient and movement with the most SD. The patient continued to use MHP prior to beginning exercises because she felt the MHP helped to decrease pain and increase her mobility. The treatment protocol was MHP, RNS with horizontal adduction in standing, l's, Y's, T's in a prone position on two of the days, push up plus on a BOSU ball on one day, while the patient also continued to stretch the pectoralis muscles in a doorway as she had been doing daily.

The clinician first applied an anterior to posterior stimulation for RNS to various places on the anterior aspect of the patient's body (i.e., upper $1 / 3$ of the sternum, middle of the sternum, xiphoid process, upper abdomen, lower abdomen, and bilateral ASIS) as the patient was standing and performing horizontal adduction. While the clinician applied the anterior to posterior force via hand pressure, the patient was instructed to close her eyes and not allow the clinician to push her backwards. The response the clinician was testing for was the largest decrease in the SD during one repetition of horizontal adduction with the external stimulation. Once the location of pressure that was the most responsive in decreasing SD the patient was found, she was asked to continue to 
keep her eyes closed, to react to the stimulus without anticipation, and told to not let the clinician push her backwards. Once the patient reacted, she then performed the active horizontal adduction while the stimulus was sustained (Figure 5).

On the first day of treatment with RNS, the patient performed two sets of ten repetitions with the pressure in the middle of her sternum. A third set was completed with the patient closing her eyes and imagining the pressure on her chest before completing the movement. When the patient imagined the pressure, the elimination of dyskinesis was consistent with the clinician applied force. Days two and three of RNS consisted of the same treatment, but on these days, the patient performed one set of ten repetitions with clinician generated force, while the second and third sets were done with the imagination of the pressure. The NPRS was collected pre and post each treatment, PSFS was collected pretreatment, and the DPA Scale was collected at day one, at discharge (day three), and eleven-months post-discharge. The patient denied taking any medications for pain and maintained her activity level throughout the course of the new treatment protocol. The patient was treated two consecutive days, then 6 days later for the third treatment. She responded well to treatment without increased pain during and after treatments. The patient did not return to the clinic until 6 days following the third treatment. At this time, the patient reported near resolution of symptoms, was able to swim throughout an entire practice without pain returning, had an improved physical exam (e.g., no tender points, improved scapular positioning, negative special tests), and met established discharge criteria. Discharge criteria had been previously established as the ability to maintain normal scapular stabilization throughout functional movements (without RNS), an average NPRS score of one out of ten or below, ${ }^{22}$ and a PSFS of a nine out of ten or higher ${ }^{23}$ with

Copyright ( $)$ by Indiana State University All rights reserved. ISSN Online 2577-8188

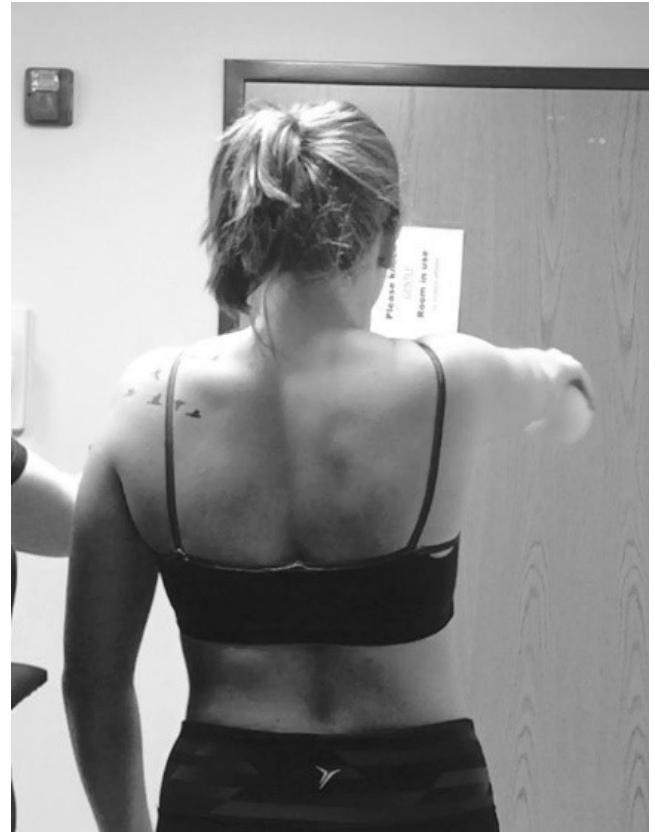

Figure 5. Scapular Dyskinesis was Eliminated when Pressure was Applied to Mid Sternum

intercollegiate swimming and conditioning activity.

\section{RESULTS}

Prior to using RNS as a treatment, the patient had received 26 days of treatment over six weeks without substantial or lasting improvements then after just three treatment sessions with RNS involved in the treatment protocol the patient met established discharge criteria (Table 3 and Table 4). At this time, a full re-evaluation was performed, intake data was collected, and no treatment was performed. The physical exam revealed the scapular slide test was equal bilaterally. The patient's primary chest breathing pattern was still present in a seated position, but diaphragm activation was now present measured through modified MARM test. The TPs on the insertion of the rhomboid major and insertion, muscle belly of the middle trapezius, and eleventh and twelfth ribs were no longer present during palpation. The TP at the middle portion of the rhomboid major was still present, but the patient reported tenderness to be mild $(2 / 10)$ Volume 2 - Issue 3 - November 2019 
Table 3. Patient Reported Outcomes Prior to RNS with Horizontal Adduction

\begin{tabular}{lccc}
\hline & Tx Day 1 & Tx Day 11 & Tx Day 21 \\
\hline NPRS - Current & $\mathbf{8}$ & $\mathbf{7}$ & 4 \\
NPRS - Best & 5 & 7 & 4 \\
NPRS - Worst & 8 & 7 & 5 \\
NPRS - Average & 7 & 7 & 4.33 \\
NPRS - Post & 6 & 6 & 3 \\
DPA Scale & 28 & $15^{*}$ & - \\
PSFS & 7.75 & 7 & 7.6 \\
\hline
\end{tabular}

*Clinically significant difference; Abbreviations: DPA Scale- Disability of the Physically Active Scale; PSFSPatient Specific Functional Scale ( $0=$ unable to perform, $10=$ fully able to perform); NPRS- Numeric Pain Rating Scale at current, best within past 24 hours, worst within last 24 hours, average of current, best and worst $(0=$ no pain, $10=$ worst pain); Pre-Tx: Pre-treatment; Post-Tx. Post-treatment; N/A: Not applicable

Table 4. Outcome Measurements After RNS with Horizontal Assuction

\begin{tabular}{ccccccc}
\hline & RNS Tx 1 & RNS Tx 2 & RNS Tx 3 & 6 day F/U & 2 wk F/U & 11 mon F/U \\
\hline DPA Scale & 22 & N/A & 16 & N/A & N/A & 4 \\
PSFS & 8 & N/A & 7 & $9.5^{*}$ & N/A & 10 \\
NPRS- Current Pre-Tx & 4 & 4 & 4 & 1 & 2 & 0 \\
NPRS-Current Post Tx & 2 & 3 & 2 & N/A & N/A & N/A \\
NPRS- Change & $2^{*}$ & $1^{*}$ & $2^{*}$ & N/A & N/A & N/A \\
Scapular Slide Test- & $1.3 \mathrm{~cm}$ & N/A & N/A & N/A & $0 \mathrm{~cm}$ & $0 \mathrm{~cm}$ \\
Horizontal Adduction & & &
\end{tabular}

*Clinically Significant Difference; DPA Scale- Disability of the Physically Active Scale; PSFS- Patient Specific Functional Scale $(0=$ unable to perform, $10=$ fully able to perform); NPRS- Numeric Pain Rating Scale at current, best within past 24 hours, worst within last 24 hours, average of current, best and worst $(0=$ no pain, 10=worst pain); Pre-Tx: Pre-treatment. Post-Tx. Post-treatment; Scapular Slide Test- Horizontal Adduction: Number is different between affected and unaffected; N/A: Not applicable

compared to initial measures (4/10). The patient also continued to experience tenderness at the first and second ribs. The patient's natural sitting posture was still forward head, forward shoulder, and increased kyphosis; however, these postures were not as noticeable as those found during the initial exam. The patient also reported it was easier to maintain good posture, and she could now do so without experiencing pain. Additionally, the previous SD that was noted with flexion, abduction, horizontal adduction, and in a push up plus position was no longer present.

Based on the physical exam and patient outcome findings, the patient was released to full activity (i.e., swimming, dryland training, and weight lifting) without further treatment, but was monitored throughout the remainder of the swim season. Follow-up measurements were collected

Copyright $($ ) by Indiana State University All rights reserved. ISSN Online 2577-8188 at 2 weeks and 11 months post-discharge (Table 4).

Detailed evaluation of patient outcomes utilizing RNS treatment revealed the patient demonstrated a change in pain that met the minimal clinically important difference (MCID) on the NPRS after Day 1, but it took 3 visits for this change to be maintained between treatment sessions (Table 4). ${ }^{24}$ Functional improvement followed a similar pattern based on PSFS scores. The patient did not report sustained functional improvement until after the third treatment. However, once this was reached, the patient retained her functional improvement all the way through the 11 month post-discharge follow-up. Additionally, the patient's scapular winging improved. At the initial exam, the patient 
displayed a $1.3 \mathrm{~cm}$ difference side to side of scapular winging with horizontal adduction; at discharge, the patient had an even distance from medial border of the scapula to spinous process with active horizontal adduction (Table 1). As with the other measures, this improvement was maintained at the 11 -month follow-up visit.

\section{DISCUSSION}

Rehabilitation for SD commonly targets the decreased activation of the serratus anterior and middle trapezius. ${ }^{25}$ Worsley et al. ${ }^{26}$ used a general rehabilitation program to retrain scapular stabilizers over a course of ten weeks. The researchers found the serratus anterior and lower trapezius could successfully be retrained over ten weeks, and scapular motion was nearly equal to a healthy population after the protocol was completed. ${ }^{26}$ The long-term benefits of this program are unknown as data was collected prior to intervention and immediately post the ten week protocol. 26

In this case study, the initial focus was on relieving pain through soft tissue treatments, and improving scapular motion through increased strength and muscle activation of the parascapular musculature. After minimal improvement, the intervention shifted towards restoring optimal movement patterns of the scapula using a reflexive neuromuscular approach. Unlike exercise-based therapy, RNS may be beneficial from an evaluative and treatment standpoint because of the immediate restoration of a functional movement pattern when the clinician applies an external force. ${ }^{27}$ If the functional movement pattern is not restored, either the wrong force is being applied (e.g., not enough force, wrong location) or RNS is not indicated.9,27 The location or amount of force may vary from patient to patient; though, when indicated, the treatment should produce an instantaneous, noticeable, and long-lasting improvement in movement. ${ }^{27}$
The proposed theories behind the success of RNS are based on influencing the central nervous system (CNS) with subconscious and reflexive movement which restores motor control and dynamic stability. Reflexive Neuromuscular Stabilization was derived from the term RNT, which was first proposed by Voight and Cook. ${ }^{28}$ The primary objective of the treatment is to trigger the subconscious process of recruiting the appropriate musculature to establish a proper movement pattern(s).8,10,28

When using an RNS treatment, the external stimulus is provided in varying forces at varying times throughout a dysfunctional movement. With RNS, the goal remains to recruit the CNS to establish appropriate recruitment strategies of involved musculature in particular movements or activities by reflexively re-patterning the neuromuscular system. In this case study, the treatment was applied to the sternum with a varying amount of force and frequency, requiring the patient to react more reflexively to an unexpected stimulus, as opposed to anticipating a consistent resistance, which helps the reaction to become subconscious. Borsa et al. ${ }^{29}$ also explained when a motion or stimulus is repeated, the brain stores these movements or stimuli, and then has the ability to access the response unconsciously. Through applying RNS, restoring the functional movement, then having the patient go through the functional movement over a number of repetitions that functional movement can now be maintained without conscious thought.

Currently, there are no published studies or case reports on utilizing RNS for SD; however, there are published reports on the use of RNT or RNS to address other dysfunction or pathology. The ability to functionally complete a previously poor motor pattern without conscious thought was elicited in a case study using RNT for apparent hamstring tightness. ${ }^{27}$ The patient in this case was tested on a variety of hamstring extensibility measurements and was classified with hamstring 
tissue extensibility dysfunction (TED). After one treatment of RNT during multi-segmental flexion, for the patient experienced substantial improvements in all ROM measurements immediately following the intervention. Not only were the gains in all ROM testing improved, but the gains in motion were enough to be within the normative ranges for each ROM measurement tested after a single intervention. ${ }^{27}$ The patient also maintained the improvements, which exceeded the gains expected from the stretching literature, at the five-week follow-up without further intervention. ${ }^{27}$ Similarly, in another case study, RNS was used in conjunction with the Mulligan Concept for a young patient with multidirectional instability of the shoulder, who had recently sustained a subluxation. ${ }^{11}$ In this case, the clinician performed a Mulligan Concept mobilization with movement followed by RNS to restore full pain-free ROM after one visit. The patient was discharged in 6 visits, returned to football activities, and did not suffer a re-injury during that season. ${ }^{11}$ Additionally, RNT has been reported to be beneficial in a case report on Anterior Cruciate Ligament $(A C L)$ deficiency. ${ }^{28}$ Over the course of eight visits, the patient experienced a large increase in strength that could only be explained by neuromuscular adaptations as opposed to true strength increases. ${ }^{28}$ Traditional strength gains require several weeks to occur, whereas neuromuscular adaptations within the body occur during the first six weeks of training. ${ }^{28}$ Further, when movement patterns changes, or the abolition of patient complaint, occur immediately with RNS application during a treatment session, it seems most likely that the mechanism of action is drive by a neurological change. Thus, of the intreatment session changes and the overall improvement over eight treatment sessions, indicate rapid neuromuscular adaptations as opposed to traditional drivers of strength improvements from exercise. ${ }^{28}$ While these three cases suggest RNS may be an effective intervention, reports on the effectiveness of RNS on SD could not be identified in the literature.

Copyright $(\subset$ by Indiana State University All rights reserved. ISSN Online 2577-8188
In the current case, applying RNS required a contraction of anterior chain muscles, in particular the abdominal muscles, to initiate stabilization before the patient tried to move the arm. The reflexive contraction, and subsequent stabilization, corrected faulty stabilization patterns allowing for more ideal functional movements (e.g., shoulder horizontal adduction). The serratus anterior and lower trapezius are typically the muscles not activating appropriately with SD. 6 It is hypothesized that activating a reflexive stabilization pattern (e.g., spinal stabilization) with RNS created the proper stability for the serratus anterior and lower trapezius to be properly coordinated to stabilize the scapula properly. Throughout the three days of treatment with RNS, the application of RNS immediately produced improvement (e.g., pain, scapular positioning), but it took until after the third treatment for the motor pattern to become ingrained enough for the patient to maintain her improvement during and after activity.

Due to the paucity of research on RNS, the ideal treatment parameters (e.g., number of treatments, sets, repetitions) required for the best treatment results are unknown. In addition, ideal locations for stimulus during RNS, or any variance across different areas of the body, functional movements (e.g., are complex movement patterns more difficult to restore), or pathology are also unknown. It is quite possible that different pathologies or movement patterns will require different parameters (e.g., differences in treatment time, frequency, duration) to produce effective patient outcomes. For example, multisegmental flexion, a uniplanar motion, required only one treatment to produce maintained resolution of a patient who present with a hamstring TED. ${ }^{27}$ In contrast, a patient with a deficient $A C L$ completed eight days of treatment with RNT, and may have needed more, to fully resolve the patient's condition. In this case, the patient did not only have one dysfunctional motion, but instead needed to restore functional movement through multiple complex movement 
patterns. Though the motions that needed restoration were complex, only three treatments of RNS were necessary for a case of SD to meet discharge criteria and maintain these improvements at 11 -month follow-up (Table 4).

The presented case provides preliminary evidence that RNS may be an effective treatment option, at least as an adjunct therapy, for patients with SD. Future research needs to be completed on RNS to determine the effectiveness of the treatment as an adjunct or individual treatment. Additionally, future research should be performed to determine the appropriate dosage of RNS to restore and maintain functional movement patterns.

\section{CLINICAL BOTTOM LINE}

The results of this case study provide initial evidence of the potential benefit of utilizing RNS. The reflexive nature of RNS, and the description of its use in this case study, may help clinicians utilize the technique to inform their clinical decision-making in determining when or how to use RNS or RNT in therapeutic rehabilitative programs. In this case, after adjusting the treatment protocol to include RNS as the primary intervention, the patient reported clinically significant improvement in pain and function. The patient reported clinically significant improvement and did not believe she needed additional treatment after three days of RNS treatment. She remained fully functional and with reduced pain 2 weeks and 11 -months post conclusion of the treatment after a multi-modal conservative rehabilitation program had failed to produce meaningful improvement over 6 weeks. Based on these results, further research on the use of RNS with SD is warranted to determine effectiveness, but this case may serve as a clinical guide for incorporating RNS into patient care.

\section{REFERENCES}

Copyright (C) by Indiana State University
1. Kibler WB. The role of the scapula in athletic shoulder function. Am J Sports Med. 1998;26(2):325-337. https://doi.org/10.1 177/03635465980 260022801.

2. Kibler WB, Ludewig PM, McClure PW, Michener LA, Bak K, Sciascia AD. Clinical implications of scapular dyskinesis in shoulder injury: The 2013 consensus statement from the 'scapular summit'. Br J Sports Med. 2013;47:877-885. https://doi.org/10.1136/bjsports-2013092425.

3. Madsen PH, Bak K, Jensen S, Welter U. Training induces scapular dyskinesis in pain-free competitive swimmers: $A$ reliability and observational study. Clin J Sport Med. 2011;21:109-113. https://doi.org/10.1097/JSM.0b013e31 $82041 \mathrm{de} 0$.

4. Martin RM, Fish DE. Scapular winging: Anatomical review, diagnosis, and treatments. Curr Rev Musculoskelet Med. $2008 ; 1(1): 1-11$. https://doi.org/10.1007/s12178-0079000-5.

5. Merolla G, De Santis E, Campi F, Paladini $P$, Porcellini $G$. Supraspinatus and infraspinatus weakness in overhead athletes with scapular dyskinesis: Strength assessment before and after restoration of scapular musculature balance.

Musculoskelet Surg. 2010;94:1 19-125. https://doi.org/10.1007/s12306-0100082-7.

6. Huang TS, Huang HY, Wang TG, Tsai YS, Lin JJ. Comprehensive classification test of scapular dyskinesis: A reliability study. Man Ther. 2015;20:427-432. https://doi.org/10.1016/i.math.2014.10 .017.

7. Burkhart SS, Morgan CD, Kibler WB. The disabled throwing shoulder: Spectrum of pathology part III: The SICK scapula, scapular dyskinesis, the kinetic chain, and rehabilitation. Arthroscopy.

Clinical Practice in Athletic Training Volume 2 - Issue 3 - November 2019 
$2003 ; 19(6): 641-661$.

https://doi.org10.1016/s07498063(03)00389-x.

8. Voight ML, Cook G. Clinical application of closed kinetic chain exercise. J Sport

Rehabil. 1996;5:25-44.

https://doi.org/10.1123/isr.5.1.25

9. Cook G. Movement: Functional movement systems: Screening, assessment, and corrective strategies. Aptos: On Target; 2010.

10. Guido JA, Stemm J. Reactive neuromuscular training: A multi-level approach to rehabilitation of the unstable shoulder. N Am J Sports Phys Ther. 2007;2(2):97-103.

1 1. Hudson RA, Baker RT, Nasypany A, Reordan D. Treatment of anterior shoulder subluxation using the mulligan concept and reflex neuromuscular stabilization: A case report. Int I Sports Phys Ther. 2017;12(1):155-162.

12. McCaffery $M$, Beebe A. Pain: Clinical manual for nursing practice. J Pain Symptom Manage. 1990;5(5):338-339. https://doi.org/10.1016/08853924(90)90052-L.

13. Vela LI, Denegar C. Transient disablement in the physically active with musculoskeletal injuries, part I: A descriptive model. J Athl Train. 2010;46(6):615-629. https://doi.org/10.4085/1062-605045.6.615.

14. Vela LI, Denegar C. The disablement in the physically active scale, part II: The psychometric properties of an outcomes scale for musculoskeletal injuries. J Athl Train. 2010;45(6):630-641. https://doi.org/10.4085/1062-605045.6.630.

15. Stratford P, Gill C, Westaway M, Binkley J. Assessing disability and change on indicidual patients: A report of a patient specific measure. Physiotherapy Canada.

Copyright (C) by Indiana State University
$1995 ; 47: 258-263$.

https://doi.org/10.3138/ptc.47.4.258.

16. Courtney R, Van Dixhoorn J, Cohen $M$.

Evaluation of breathing pattern:

Comparison of a manual assessment of respiratory motion (MARM) and respiratory induction plethysmography. Appl Psychophysiol Biofeedback. 2008;33(2):91-100. https://doi.org/10.1007/s10484-0089052-3.

17. Chaitow L, Christopher G, Dinah B. Recognizing and Treating Breathing Disorders: A Multidisciplinary Approach. 2nd ed. Endinburgh: Churchill Livingstone; 2014.

18. Curtis T, Roush JR. The lateral scapular slide test: A reliability study of males with and without shoulder pathology. N Am J Sports Phys Ther. 2006;1 (3):140-046.

19. Ozunlu N, Tekeli H, Baltaci G. Lateral scapular slide test and scapular mobility in volleyball players. J Athl Train.

2011 ;46(4):438-444. https://doi.org/10.4085/1062-605046.4.438.

20. White MG. Breathing.com. Breathing.com. Available at: http://www.breathing.com. Accessed February 22, 2015.

21. Nasypany A. Primal Relfex Release Technique: Level 1 Training.

22. Krebs EE, Carey TS, Weinberger $M$. Accuracy of the pain numeric rating scale as a screening test in primary care. J Gen Intern Med. 2007;22(10):1453-1458. https://doi.org/10.1007/s1 1606-0070321-2.

23. Horn $K H$, Jennings $S$, Richardson $G$, Van Vliet D, Hefford C, Abbott JH. The patient-specific functional scale: Psychometrics, clinimetrics, and application as a clinical outcome measure. $J$ Orthop Sports Phys Ther. 201 2;42(1):30-42. https://doi.org/10.2519/iospt.2012.372 7. 
24. Hefford C, Abbott JH, Arnold R, Baxter GD. The patient-specific functional scale: Validity, reliability, and responsiveness in patients with upper extremity musculoskeletal problems. J Orthop Sports Phys Ther. 2012;42(2):56-65. https://doi.org/10.2519/iospt.2012.395 3.

25. Huang TS, Ou HL, Huang CY, Lin JJ. Specific kinematics and associated muscle activation in indiciduals with scapular dyskinesis. J Shoulder Elbow Surg. 1995;24:1 227-1234. https://doi.org/10.1016/i.jse.2014.12.0 $\underline{22}$.

26. Worsley P, Warner M, Mottra S, et al. Motor control retraining exercises for shoulder impingement: Effects on function, muscle activation, and biomechanics in young adults. J Shoulder Elbow Surg. 2013;22:e1 1-el9. https://doi.org/10.1016/i.jse.2012.06.0 10.

27. Loutsch RA, Baker RT, May JM, Nasypany AM. Reactive neuromuscular training results in immediate and long term improvements in measures of hamstring flexibility: A case report. Int I Sports Phys Ther. 2015;10(3):371-377.

28. Cook G, Burton L, Fields K. Reactive neuromuscular training for the anterior cruciate ligament-deficient knee: A case report. J Athl Train. 1999;34(2):194-201.

29. Borsa PA, Lephart SM, Kocher MS, Lephart SP. Functional assessment and rehabilitation of shoulder proprioception for glenohumeral instability. J Sport Rehabil. 1994;3:84-104. https://doi.org/10.1123/isr.3.1.84. 